\title{
Renin and Lysosomal Enzyme Activities in the Kidney of Spontaneously Hypertensive Rats with Accelerated Hypertension
}

\author{
Noboru Saito, Sakae Mukaino, and Koichi Ogino
}

We observed increased activities of cathepsin and $\beta$-glucuronidase in the kidney of rats with DOGA hypertension (Saito et al : Jap Circulat J 37 : 1277, 1973). The present study was undertaken to clarify the relationship between renin or lysosomal enzymes and renal vascular lesions. Four- or 10-month-old male stroke-prone SHR (SHRSP), stroke-resistant SHR (SHRSR), and Wistar Kyoto (WK) rats were used in experiment 1. In experiment 2, 4-month-old male SHR and WK rats were given a high salt chow containing $7 \% \mathrm{NaCl}$ and $0.9 \%$ saline. The control SHR and $\mathrm{WK}$ rats were given a normal chow containing $0.6 \% \mathrm{NaCl}$ and tap water.

In experimental procedure (a), rats were given high salt chow and saline for 18 days, then normal chow and tap water for 13 days, again high salt chow and tap water for 17 days and finally high salt chow and saline for 3 days. In experimental procedure (b), rats were given normal chow and tap water for 41 days after the experimental procedure (a). The control rats were given normal chow and tap water during whole period.

Systolic blood pressure of the rat was determined by the plethysmographic method. Rats were exanguinated by decapitation or by ether anesthesia. The rat kidney removed was homogenized, using Potter-Elvehjem homogenizer with $0.45 \mathrm{M}$ sucrose $(1: 8 \mathrm{w} / \mathrm{v})$. The cytoplasmic fraction of the rat kidney was obtained by centrifuging the homogenate at $650 \mathrm{G}$ for $2.5 \mathrm{~min}$ and was used for the assay of enzymes after 5 times freezing and thawing. The method of enzyme assay was described previously (Saito et al: Jap Circulat J $37: 1277,1973$ ). For the assay of $\beta$-N-acetylglucosaminidase $\left(\beta\right.$-NAGA), incubation was performed at $37^{\circ} \mathrm{C}$ for $20 \mathrm{~min}$ at $\mathrm{PH} 4.4$. Protein concentration was assayed by the method of Lowry et al.

In experiment 1 , SHRSP aged 4 months or 10 months showed blood pressure of $206 \pm 8 \mathrm{mmHg}(\mathrm{M} \pm \mathrm{SD})$ or $206 \pm 17 \mathrm{mmHg}$ and the control $\mathrm{WK}$ rats showed that of $112 \pm 10 \mathrm{mmHg}$ or $116 \pm 10 \mathrm{mmHg}$, respectively. SHRSR aged 10 months showed $181 \pm 17 \mathrm{mmHg}$. The body weight of 10 -month-old SHRSP was less than that of SHRSR or WK rats, though that of 4-month-

From the Clinical Nutrition Laboratory, and Third Division, Department of Internal Medicine, Faculty of Medicine, Kyoto University, Kyoto. 
Vol. 16
No. 3

SPONTANEOUSLY HYPERTENSIVE RAT

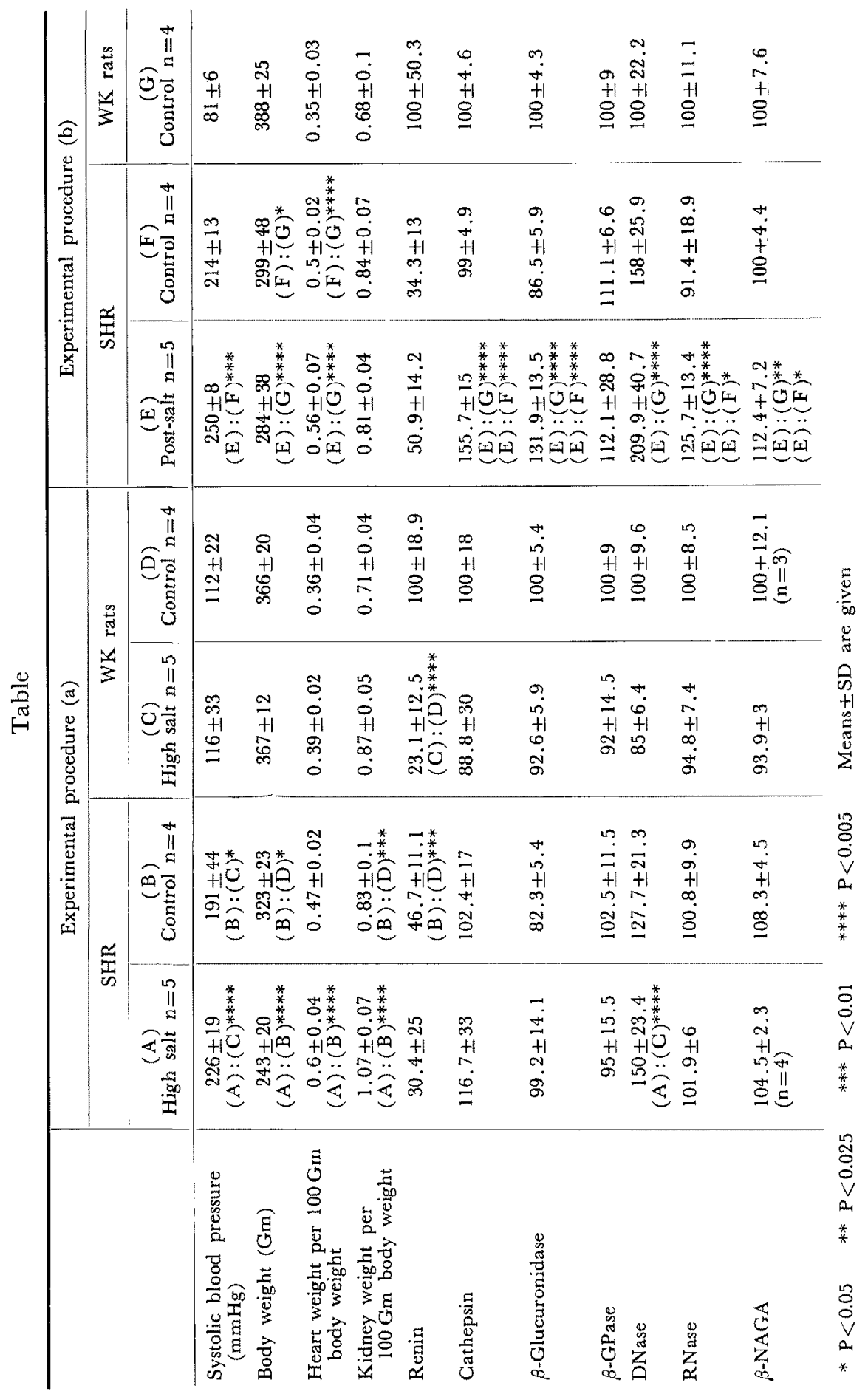


old SHRSP was almost equal to that of WK rats. Kidney renin activity (KRA) of SHR was less than KRA of the control WK rat. But KRA was more in 10-month-old SHRSP than in SHRSR of the same age. Activities of $\beta$-glucuronidase, deoxyribonuclease (DNase) and ribonuclease (RNase) were higher in 4-month-old SHRSP than in WK rats and the same tendency was also shown in 10-month-old rats except for in $\beta$-glucuronidase. DNase and $\beta$-NAGA activities of SHRSR were higher than those of WK rats.

The activity of $\beta$-glycerophosphatase ( $\beta$-GPase) showed no significant difference among 3 groups. In experimental procedure (a) of experiment 2 , angionecrosis of renal arterioles and small arteries was shown microscopically in SHR which were given high salt diet. The values of enzyme activities were shown in Table in which control values were expressed as 100 . Under salt loading, DNase activity of SHR was higher than that of WK rats. KRA of SHR was less than KRA of WK rats in the control groups. KRA of SHR was higher in average than KRA of WK rats under salt loading, but the difference between them was not significant. In experimental procedure (b), SHR pretreated with high salt (post-salt SHR) showed the highest blood pressure and severe vascular damages. Cathepsin, $\beta$-glucuronidase, RNase, and $\beta$-NAGA activities of post-salt SHR were higher than those of the control SHR or WK rats (Table). DNase activity of post-salt SHR was higher than that of the control WK rats. KRA of post-salt SHR was higher in average than KRA of the control SHR, but the difference between them was not significant. Okamoto et al recently succeeded in separation of SHRSP from SHRSR. SHRSP and post-salt SHR tend to have accelerated hypertension. Plasma renin concentration of SHRSP was higher than that of WK rat in 10 months of age (Saito et al : Jap Circulat J, in press), while KRA of SHRSP was higher than KRA of SHRSR in 10 months of age. These results on renin activity may indicate that the increased renin activity of SHRSP is due to severe renal vascular lesions. Wolinsky et al reported increased lysosomes in aortic smooth muscle cells in hypertensive rats (Wolinsky et al: Am J Pathol 73: 727, 1973). Increased activities of many lysosomal enzymes in post-salt SHR may demonstrate the intimate relationship between lysosomal enzyme activities and vascular lesions.

(This research is sponsored by the Science and Technology Agency of Japanese Government.) 